\title{
Coulometry and Calorimetry of Electric Double Layer Formation in Porous Electrodes
}

\author{
Mathijs Janssen, ${ }^{1, *}$ Elian Griffioen, ${ }^{2}$ P. M. Biesheuvel, ${ }^{3}$ René van Roij, ${ }^{1}$ and Ben Erné ${ }^{2, \dagger}$ \\ ${ }^{1}$ Institute for Theoretical Physics, Center for Extreme Matter and Emergent Phenomena, \\ Utrecht University, Princetonplein 5, 3584 CC Utrecht, The Netherlands \\ ${ }^{2}$ Van 't Hoff Laboratory for Physical and Colloid Chemistry, Debye Institute for Nanomaterials Science, \\ Utrecht University, Padualaan 8, 3584 CH Utrecht, The Netherlands \\ ${ }^{3}$ Wetsus, European Centre of Excellence for Sustainable Water Technology, Oostergoweg 9, 8911 MA Leeuwarden, The Netherlands
}

(Received 30 June 2017; revised manuscript received 31 August 2017; published 19 October 2017)

Coulometric measurements on salt-water-immersed nanoporous carbon electrodes reveal, at a fixed voltage, a charge decrease with increasing temperature. During far-out-of-equilibrium charging of these electrodes, calorimetry indicates the production of both irreversible Joule heat and reversible heat, the latter being associated with entropy changes during electric double layer (EDL) formation in the nanopores. These measurements grant experimental access - for the first time-to the entropic contribution of the grand potential; for our electrodes, this amounts to roughly $25 \%$ of the total grand potential energy cost of EDL formation at large applied potentials, in contrast with point-charge model calculations that predict $100 \%$. The coulometric and calorimetric experiments show a consistent picture of the role of heat and temperature in EDL formation and provide hitherto unused information to test against EDL models.

DOI: 10.1103/PhysRevLett.119.166002

Where surfaces of charged electrodes meet fluids that contain mobile ions, so-called electric double layers (EDLs) form that screen the electric surface charge by a diffuse cloud of counterionic charge. This EDL has been intensively studied for over a century and is of paramount importance to many processes in physical chemistry and soft matter physics. With the ongoing development of nanomaterials, nowadays electrodes can be made from porous carbon with internal surface areas exceeding $1000 \mathrm{~m}^{2} \mathrm{~g}^{-1}$. These porous electrodes can be immersed in a variety of electrolyte solutions or ionic liquids. A socalled electric double layer capacitor (EDLC) is then formed, whose high capacitance makes it a prime candidate for capacitive energy storage, energy conversion [1,2], and water desalination [3-5]. In these porous electrodes, solvated ions have a size similar to that of their confining geometry; hence, a realistic theory must at least address both the electrostatics and the packing of the ions. Simulations and in situ analytical techniques [6] have revealed a wealth of phenomena in EDLCs [7], including overscreening [8], ion desolvation [9,10], in-plane structural transitions [11], layered packings of counterionic charge at high surface potentials [12], and, relatedly, oscillations in the EDL capacitance with decreasing pore width [13-15]. Unfortunately, the gap between (computationally demanding) first-principles models and experimental measurements on the charging behavior of porous electrodes is far from closed, with many questions remaining regarding the precise screening mechanisms at play [16]. While our understanding of the EDL is based mainly on isothermal numerical and experimental methods, recent work has revealed an interplay between temperature, heat, and entropy in the EDL. In particular, both model calculations [17] and experiments [2] indicate that the surface potential of an electrode with a fixed high surface charge should rise by about $1 \mathrm{mV} \mathrm{K}^{-1}$ with increasing temperature. Conversely, EDL formation under adiabatic settings induces a thermal response that has been largely overlooked [18]. During charging, ions and solvent molecules order into the EDL; hence, the configurational contribution to the total phase-space volume decreases. During isentropic charging, this decrease must be balanced by an equal and opposite increase in the momentum contribution: the electrolyte solution heats up. As the source of reversible temperature variations is nonzero only within the EDL, temperature variations scale inversely with the average electrode pore size. The development of high-surface-tovolume-ratio electrodes (for, e.g., supercapacitors) was therefore required before the small temperature variations were detected experimentally [19].

The temperature-dependent phenomena mentioned above are (in principle) measurable and therefore open new possibilities for experiments against which EDL theories can be tested. In this Letter on the temperaturedependent EDL we present data of two experiments involving water-immersed porous carbon electrodes, which is an important system for water desalination and blueenergy devices [1]. Conceptually, the two experiments are each other's "opposites": while the first experiment involves a temperature-induced charge variation, the second experiment studies a charge-induced temperature variation. More specifically, with a potentiostatic coulometry experiment we determine the temperature dependence of the equilibrium charge of our blocking electrodes at 
fixed potential. Two thermodynamic identities then allow us to predict not only the heat required to flow out of the cell upon isothermal charging, but also the thermal response to adiabatic charging. In the second, calorimetric experiment, we probe the thermal response of the capacitor to far-from-equilibrium charging. We then distinguish irreversible Joule heat from reversible heat, which we identify with the entropic contribution to the grand potential. While previous work built theoretical models for the latter quantity [20,21], to our knowledge, this Letter presents its first experimental determination.

Experiments were performed using a homebuilt electrochemical cell submerged in a thermostatic water bath maintained at $25^{\circ} \mathrm{C}$ (Julabo F25). The cell, depicted in Fig. 1(a), had in- and outlets for a degassed aqueous $\mathrm{NaCl}$ solution at salt concentration $\rho_{\mathrm{s}}$ and a Pt100 temperature probe $(0.5 \mathrm{~mm}$ diameter, miniature RTD sensor from TC Direct) that measured the temperature $T_{1}$ within the cell. The tip of the probe was centered halfway between two concentric and parallel electrodes, separated from each other by $2.2 \mathrm{~mm}$. The electrodes were disks of $25 \mathrm{~mm}$ in diameter cut from sheets of porous carbon: $0.5 \mathrm{~mm}$ thick, with a density of $0.58 \mathrm{gm} \mathrm{L}^{-1}$, a porosity of $65 \%$, weighing $0.18 \mathrm{~g}$ each, and a Brunauer-Emmett-Teller area of $1330 \mathrm{~m}^{2} \mathrm{~g}^{-1}$ from nitrogen adsorption [4]. These disks were glued with conductive silver epoxy onto graphite current collector disks. In turn, the electrodes and current collectors were mounted in a glass casing, with holes

(a)

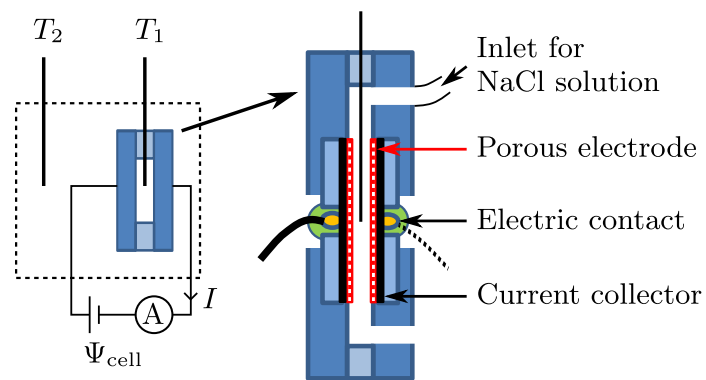

(b)

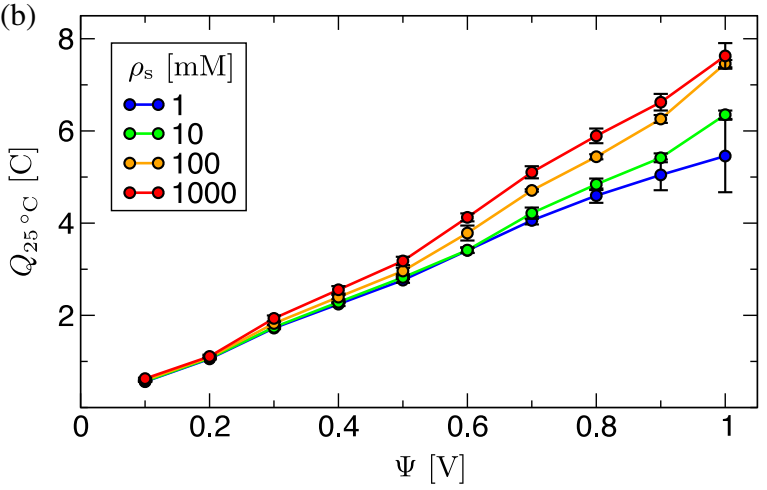

FIG. 1. (a) The experimental setup to measure temperature and charge during (dis)charging of porous carbon electrodes $(0.18 \mathrm{~g}$ each) in $\mathrm{NaCl}$ solution. (b) The dependence of the total charge $Q$ on $\Psi$ is shown at $\rho_{\mathrm{s}}=1,10,100,1000 \mathrm{mM}$. Error bars are based on two or more duplicate measurements. allowing copper wires to make electric contact with both collectors using silver epoxy glue. There, nonconducting glue applied onto the copper and silver epoxy prevented direct contact with the thermostatic water bath. Cell voltages were applied and electrical currents were measured using a potentiostat-galvanostat PGSTAT100 from Metrohm Autolab. The temperature difference $\Delta T=T_{1}-$ $T_{2}$ was measured compared to a second Pt100 temperature probe $\left(T_{2}\right)$ immersed directly in the thermostatic bath. Without an applied potential, $\Delta T$ was constant within $0.007^{\circ} \mathrm{C}$ for several days.

In both experiments, at time $t=t_{0}$ the cell voltage $\Psi_{\text {cell }}$ was changed stepwise from $0 \mathrm{~V}$ to different maximum values $\Psi$ not exceeding $1 \mathrm{~V}$. The total charge $Q_{T_{1}}(\Psi)=$ $\int_{t_{0}}^{t_{1}} I d t$ on the electrodes' surface at temperature $T_{1}$, shown for several salt concentrations in Fig. 1(b), was found by integrating the current $I$ until $t_{1}, 4$ hours later.

In the coulometric experiment [see Fig. 2(a)], after the voltage rise from $0 \mathrm{~V}$ to $\Psi$ at $t_{0}$ and approximately 4 hours of subsequent equilibration (all at $25.3 \pm 0.1^{\circ} \mathrm{C}$ ), the temperature was alternated at fixed voltage $\Psi$ between two temperatures differing by $\Delta T_{\text {step }}$, with ample time for equilibration in between. Here, the temperature changeswith a temporal spacing of 2 hours - are accomplished at a thermal sweeping rate of $10^{\circ} \mathrm{C}$ per hour. By time integrating peaks in the current $I$, we determined the excess charge $\Delta Q$ that flowed because of the temperature-induced change in the capacitance. For instance, at $\Psi=1 \mathrm{~V}$ and $\Delta T_{\text {step }}=2.5^{\circ} \mathrm{C}$, the charge decreased from $Q_{25.3^{\circ} \mathrm{C}}=$ $8.11 \mathrm{C}$ by an amount of $\Delta Q=Q_{27.8{ }^{\circ} \mathrm{C}}-Q_{25.3^{\circ} \mathrm{C}}=$ $-9.40 \mathrm{mC}$, i.e., a relative decrease of about $0.05 \% \mathrm{~K}^{-1}$. The charge decrease scaled to the temperature step is shown in Fig. 2(b) for several $\Delta T_{\text {step }}$ and $\Psi$. We see that $\Delta Q$ scales linearly with both $\Delta T_{\text {step }}$ and $\Psi$. We write $(\partial \Delta Q / \partial T)_{\Psi}=\alpha \Psi$, and determine $\alpha=-4.02 \pm$ $0.07 \mathrm{mC} \mathrm{V}^{-1} \mathrm{~K}^{-1}$ from a linear fit to the data in Fig. 2(b).
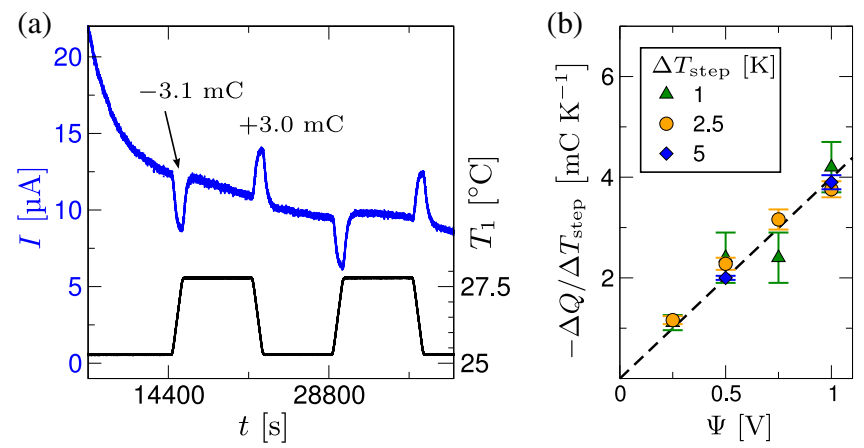

FIG. 2. (a) In the coulometric experiment, the current (blue) initially exhibits a monotonic decay, onto which positive and negative peaks are superimposed when the electrolyte temperature (black) is decreased and increased. Plotted are data for $\Delta T_{\text {step }}=2.5^{\circ} \mathrm{C}, \rho_{\mathrm{s}}=1 \mathrm{M}$, and $\Psi=0.25 \mathrm{~V}$. (b) The temperature-scaled equilibrium charge difference $\Delta Q / \Delta T_{\text {step }}$ is shown at different potential and temperature steps. 
With $\alpha$ at hand, we can predict the heat $\mathbb{Q}$ required to flow into the device upon isothermal charging, and also the adiabatic temperature rise $\Delta T_{\text {adiab }}$ upon charging the cell, had it been thermally insulated. The required thermodynamic identities for $\mathbb{Q}$ and $\Delta T_{\text {adiab }}$ were previously derived in Refs. [2,17], respectively, and take simplified forms for the capacitor of interest [22]. In particular, the isothermal heat amounts to $\mathbb{Q}=\alpha T \Psi^{2} / 2$; hence, $\mathbb{Q}=-0.60 \pm 0.01 \mathrm{~J}$ at $\Psi=1 \mathrm{~V}$; i.e., a positive amount of heat flows out of the capacitor during isothermal charging, while an equal amount of heat should flow into the capacitor during the mirror discharging process. In the presence of insulating walls, heat cannot exit the cell and the capacitor would exhibit a temperature rise of $\Delta T_{\text {adiab }}=$ $\alpha T \Psi^{2} /\left(2 \mathbb{C}_{p}\right)$ upon charging-with $\mathbb{C}_{p}=10.9 \pm 0.1 \mathrm{~J} \mathrm{~K}^{-1}$ being the heat capacity of the cell [22] —and an equal-sized cooling effect for the mirror discharging process. At $\Psi=1 \mathrm{~V}$, this amounts to $\Delta T_{\text {adiab }}=0.055 \pm 0.002 \mathrm{~K}$.

In the second, calorimetric, experiment, after the initial voltage rise and subsequent equilibration, at $t=t_{1}$ we switch the voltage [see Fig. 3(a)] back to 0 and, while measuring $\Delta T$, wait another 4 hours until $t=t_{2}$ when the current $I$ [Fig. 3(b)] has essentially dropped to 0 [22]. Since this (dis)charging process is far from equilibrium, the $\Delta T$ peaks in Fig. 3(c) are caused both by reversible rearrangements within the EDL and by irreversible strictly positive Joule heating. Clearly, the exothermic Joule heat dominates in both directions of charge transfer. Nevertheless, heat is more exothermic upon charging $\left(t_{0} \rightarrow t_{1}\right)$ than upon discharging $\left(t_{1} \rightarrow t_{2}\right)$; the difference is ascribed to the reversible part of the heat exchange, which is exothermic upon charging and endothermic upon discharging.

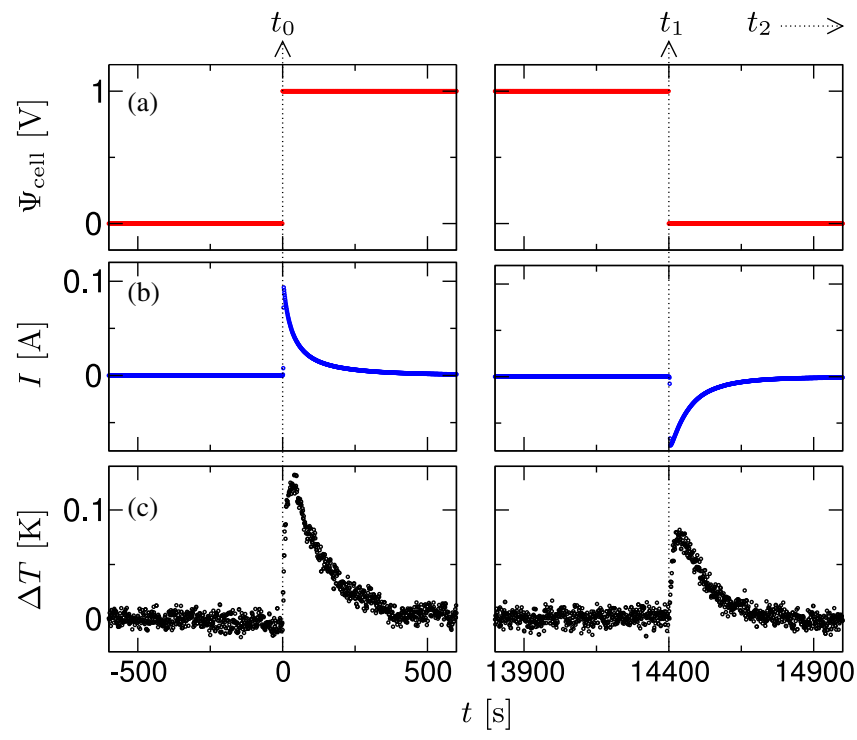

FIG. 3. In the calorimetric experiment, the cell voltage $\Psi_{\text {cell }}$ (a) was switched to $\Psi$ at $t_{0}$ and $0 \mathrm{~V}$ was applied at $t_{1}$. The current $I$ (b) and temperature difference $\Delta T$ (c) were measured simultaneously. Plotted are data for $\Psi=1 \mathrm{~V}$ and $\rho_{\mathrm{s}}=1 \mathrm{M}$.
At any moment in this charging and discharging cycle, the measured temperature difference $\Delta T$ could, in principle, be found from the heat equation $[26,27]$,

$$
\varrho c_{p} \partial_{t} T=\kappa \nabla^{2} T+\mathbf{I} \cdot \mathbf{E}
$$

describing the time evolution of the temperature $T(\mathbf{x}, t)$ at any point $\mathbf{x}$ in the thermostatic bath $\mathcal{V}_{\text {bath }}$, the solid $\mathcal{V}_{s}$ (carbon electrodes, glass casing, circuitry, etc.), and the electrolyte solution $\mathcal{V}_{\text {el }}$ regions of the cell. Here, the ionic current $\mathbf{I}$ and electric field $\mathbf{E}$ are spatially varying and (possibly) nonzero only within $\mathcal{V}_{\mathrm{el}}$. Moreover, all material properties appearing in Eq. (1) are locally defined; the specific heat capacity $c_{p}$, mass density $\varrho$, and heat conductivity $\kappa$ take different values in the different parts of the system $\left(\mathcal{V}_{\text {bath }}, \mathcal{V}_{s}, \mathcal{V}_{\mathrm{el}}\right)$. Solving Eq. (1) for the complete three-dimensional geometry of the cell (including the porous network) and bath is out of the scope of our study and a simplified analysis of the experiments is performed.

In our analysis, the temperature is assumed to be homogeneous within the complete cell, with a steep drop of $\Delta T$ between the cell and the thermostatic bath. The temperature difference $\Delta T$ between the cell and the thermostatic bath changes when heat is added at another rate than heat is lost to the environment. If the heat flow to the environment is assumed to be linear with $\Delta T$, as in Newton's law of cooling, the temperature difference $\Delta T$ is governed by

$$
\mathbb{C}_{p} \frac{d \Delta T}{d t}=-K \Delta T+\dot{\Pi}_{\mathrm{tot}},
$$

where we assume $d \Delta T / d t=d T_{1} / d t$, thanks to a practically constant $T_{2}$, and with $\dot{\Pi}_{\text {tot }}=\int_{\mathcal{V}_{\mathrm{el}}} d \mathbf{x} \mathbf{I} \cdot \mathbf{E}$ being the total heating rate in $\mathrm{J} \mathrm{s}^{-1}$, and $K$ being the heat transfer coefficient in $\mathrm{J} \mathrm{s}^{-1} \mathrm{~K}^{-1}$ of the cell-bath interface.

Equation (1) stems from an internal energy balance; the source term $\mathbf{I} \cdot \mathbf{E}$ captures the exchange between electric field energy and the solution's thermal energy as the electric field performs work on the electrolyte solution. This source term can be split up in reversible $(\sim I)$ and irreversible contributions $\left(\sim I^{2}\right)$, respectively, with only the reversible term persisting in the limit of slow charging $[18,28]$. Likewise, for the total heat production rate appearing in Eq. (2) we similarly write

$$
\int_{t_{0}}^{t_{2}} \dot{\Pi}_{\mathrm{tot}} d t \equiv \Pi_{\mathrm{tot}} \equiv \Pi_{\mathrm{rev}}^{\mathrm{ch}}+\Pi_{\mathrm{irr}}^{\mathrm{ch}}+\Pi_{\mathrm{rev}}^{\mathrm{dis}}+\Pi_{\mathrm{irr}}^{\mathrm{dis}}
$$

with reversible (rev) and irreversible (irr) heat contributions, during charging (ch, $t_{0} \leq t<t_{1}$ ) and discharging (dis, $t_{1} \leq t \leq t_{2}$ ). The right-hand side of Eq. (3) simplifies since the reversible heat during charging opposes that during discharging by definition $\left(\Pi_{\mathrm{rev}}^{\mathrm{ch}}=-\Pi_{\mathrm{rev}}^{\mathrm{ch}}\right)$. To appreciate the significance of the term $\Pi_{\mathrm{rev}}^{\mathrm{ch}}$ (and likewise, $\Pi_{\mathrm{rev}}^{\mathrm{dis}}$ ), we note that reversible work performed by the electric field 
on the electrolyte solution equals the entropic contribution $\Omega_{\text {ent }}$ to the grand potential $\Omega$, as was pointed out by Overbeek [20]: "during the charging process ... the solution part of the double layer arranges itself automatically, i.e., with zero contribution to the free energy, and thus the change in entropy and the electrical work done [on the solution, $\int_{\mathcal{V}_{\mathrm{el}}} d \mathbf{x} \int_{0}^{\psi} \psi d q$, with $\psi(\mathbf{x})$ and $q(\mathbf{x})$ being the local electrostatic potential and charge density, respectively] just compensate one another." Hence, $\Pi_{\mathrm{rev}}^{\mathrm{ch}}$ is to be identified with the entropic contribution $\Omega_{\mathrm{ent}}$ to the grand potential $\Omega$ of the cell during EDL buildup, which, as far as we know, has not previously been measured experimentally. In fact, the equality $\Pi_{\mathrm{rev}}^{\mathrm{ch}}=\Omega_{\mathrm{ent}}^{\mathrm{GC}}$ can be explicitly derived within the classical Gouy-Chapman (GC) EDL model, for which an analytical expression for $\Omega_{\text {ent }}$ is known [22].

To find $\Pi_{\mathrm{rev}}^{\mathrm{ch}}$, we first need to determine the empirical parameter $K$, which is done in Supplemental Material [22] via two calibration methods, both employing known quantities of Joule heat. One of these methods used the geometry with carbon electrodes (c.e.) as described so far, and determined $K^{\text {c.e. }}=0.239 \pm 0.003 \mathrm{~J} \mathrm{~s}^{-1} \mathrm{~K}^{-1}$ directly from the data presented in Fig. 3. In this setup, most of the Joule heat could not have been produced in the electrolyte solution, as the total resistance went up from 13 to merely $70 \Omega$ upon going from $1 \mathrm{M}$ to $1 \mathrm{mM} \mathrm{NaCl}$, despite a factor of 1000 in electrolyte conductivity. Instead, the Joule heat was probably produced at the electrical contacts between the porous carbon electrodes and the external circuit [4]. In the other calibration method, we replaced the electrodes with heating elements (h.e.) where heat is generated in a wire of known resistance. This gave $K^{\text {h.e. }}=0.179 \pm 0.001 \mathrm{~J} \mathrm{~s}^{-1} \mathrm{~K}^{-1}$. Even though the latter cell setup has a (slightly) different heat conductance and conductivity, we deem this calibration method with heating elements superior because (1) Joule heat is determined more accurately and (2) the heat with which is calibrated is generated at the same location as the reversible heat, the quantity we set out to find.

With $K$ at hand, we consider the total heat production during charging,

$$
\Pi_{\mathrm{tot}}^{\mathrm{ch}} \equiv \Pi_{\mathrm{irr}}^{\mathrm{ch}}+\Pi_{\mathrm{rev}}^{\mathrm{ch}}=K \int_{t_{0}}^{t_{1}} \Delta T(t) d t
$$

and discharging,

$$
\Pi_{\mathrm{tot}}^{\mathrm{dis}} \equiv \Pi_{\mathrm{irr}}^{\mathrm{dis}}+\Pi_{\mathrm{rev}}^{\mathrm{dis}}=K \int_{t_{1}}^{t_{2}} \Delta T(t) d t
$$

separately. Using $K=K^{\text {h.e. }}$, we plot $\Pi_{\text {tot }}^{\text {ch }}$ and $\Pi_{\text {tot }}^{\text {dis }}$ as a function of $\Psi$ for different salt concentrations in Fig. 4(a). We observe that the salt concentration has only a minor effect; results at $\rho_{\mathrm{s}}=1 \mathrm{mM}$ did not differ very much from those at $\rho_{\mathrm{s}}=1 \mathrm{M}$. The reversible heat production is now determined via

$$
\Pi_{\mathrm{rev}}^{\mathrm{ch}}=\frac{\Pi_{\mathrm{tot}}^{\mathrm{ch}}-\Pi_{\mathrm{tot}}^{\mathrm{dis}}}{2}
$$

where we used that $\Pi_{\mathrm{rev}}^{\mathrm{ch}}=-\Pi_{\mathrm{rev}}^{\mathrm{dis}}$ (by definition) and that $\Pi_{\text {irr }}^{\text {dis }}=\Pi_{\text {irr }}^{\text {ch }}($ checked independently [22]). The potential dependence of $\Pi_{\mathrm{rev}}^{\mathrm{ch}}$ is shown in Fig. 4(b). Since $\Pi_{\mathrm{rev}}^{\mathrm{ch}}$ is an equilibrium property of the EDL, it should be independent of the thermal boundary condition (insulated or not), so we could find the temperature rise upon adiabatic charging from Eq. (2) by setting $K=0$, leading at $\Psi=1 \mathrm{~V}$ to $\Delta T_{\text {adiab }}=\Pi_{\mathrm{rev}}^{\mathrm{ch}} / \mathbb{C}_{p}=0.082 \pm 0.016 \mathrm{~K}$. It is reassuring to see that the coulometric and calorimetric experiments provide comparable predictions for $\Delta T_{\text {adiab }}$ via two completely independent routes.

Identifying $\Pi_{\mathrm{rev}}^{\mathrm{ch}}=\Omega_{\mathrm{ent}}$, we have experimental access to the ratio $\Omega_{\text {ent }} / \Omega$, where, for isothermal charging processes, the grand potential $\Omega=\int \Psi d Q$ equals (minus) the electric work required to charge the cell, which we find by
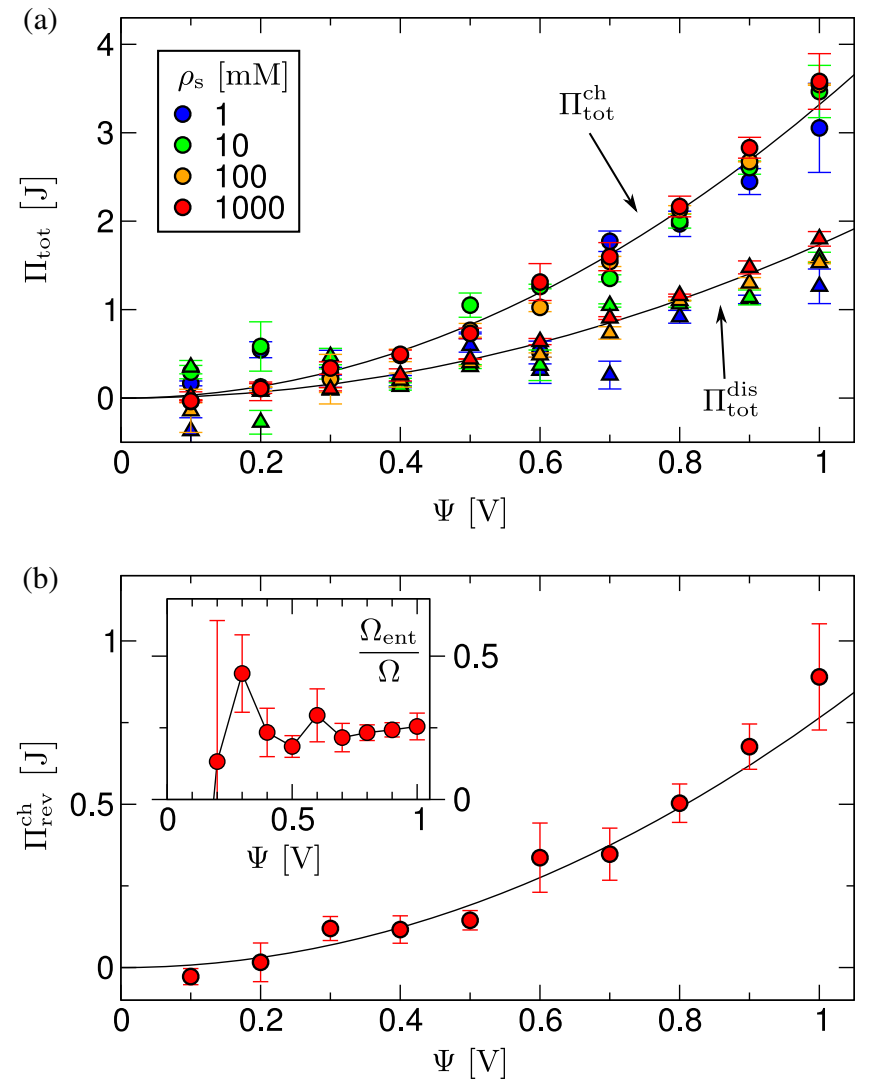

FIG. 4. (a) The total heat production during charging $\Pi_{\text {tot }}^{\text {ch }}$ (circles) and discharging $\Pi_{\text {tot }}^{\text {dis }}$ (triangles) [see Eqs. (4) and (5)] versus the cell voltage $\Psi$ at $\rho_{\mathrm{s}}=1,10,100,1000 \mathrm{mM}$. (b) The reversible heat $\Pi_{\text {rev }}^{\text {ch }}$ [Eq. (6)] versus potential was measured at $\rho_{\mathrm{s}}=1 \mathrm{M}$. Black lines in (a) and (b) indicate quadratic fits through the data as guides to the eye. Identifying $\Pi_{\mathrm{rev}}^{\mathrm{ch}}=\Omega_{\mathrm{ent}}$, the inset shows the ratio $\Omega_{\mathrm{ent}} / \Omega$ versus potential. 
integrating an interpolation through the capacitance data of Fig. 1(b). This ratio- shown in the inset of Fig. 4(b) - is approximately $25 \%$ at high potentials, while its behavior at low potential remains uncertain. This finding can be compared (qualitatively) to the predictions of different EDL models. In particular, Gouy-Chapman theory predicts $\Omega_{\text {ent }} / \Omega=1 / 2$ at low potentials, while for high potentials this ratio approaches unity [20]. This finding for pointlike ions is in stark contrast to later theoretical work that included finite ionic size [21]. There, it was reported that the electrostatic energy $\Omega_{\mathrm{el}}$ gains in relative importance at higher potentials, at the expense of a decrease in the importance of $\Omega_{\text {ent }}$. Hence, the EDL models that include ionic volume are in qualitative agreement with our experimental findings.

In this Letter on temperature effects in electric double layer capacitors we presented two different temperaturedependent experiments involving water-immersed porous carbon electrodes. Both experiments point toward exothermic heating (heat flowing out of the cell) during quasistatic EDL formation and the opposite effect during quasistatic discharging. We moreover presented the first experimental measurement of the entropic contribution to the grand potential energy cost of electric double layer formation. At high electrostatic potentials, this term constitutes approximately $25 \%$ of the total grand potential energy. The accuracy of this prediction could be improved in future work by building an even more sensitive calorimetric cell. While this first study considered only $\mathrm{NaCl}$ in water, future work could also look at the effect of ionic valency and size. The proposed calorimetric method is useful for distinguishing or ruling out theories, which is important for understanding the electric double layer in porous carbon. This is of fundamental as well as practical importance, as the potential of these materials for future energy harvesting and storage can hardly be overstated.

Henkjan Siekman and Peter de Graaf are thanked for making the electrochemical cell, and Bonny Kuipers and Stephan Zevenhuizen are thanked for technical support. This work is part of the D-ITP consortium, a program of the Netherlands Organization for Scientific Research (NWO) that is funded by the Dutch Ministry of Education, Culture and Science (OCW). R. v. R. acknowledges financial support from an NWO-VICI grant.

*Corresponding author.

mjanssen@is.mpg.de

Corresponding author.

B.H.Erne@uu.nl

[1] D. Brogioli, Phys. Rev. Lett. 103, 058501 (2009).

[2] A. Härtel, M. Janssen, D. Weingarth, V. Presser, and R. van Roij, Energy Environ. Sci. 8, 2396 (2015).

[3] A. Hemmatifar, M. Stadermann, and J. G. Santiago, J. Phys. Chem. C 119, 24681 (2015).
[4] T. Kim, J. E. Dykstra, S. Porada, A. Van Der Wal, J. Yoon, and P. M. Biesheuvel, J. Colloid Interface Sci. 446, 317 (2015).

[5] S. Rubin, M. E. Suss, P. M. Biesheuvel, and M. Bercovici, Phys. Rev. Lett. 117, 234502 (2016).

[6] H. Wang, A. C. Forse, J. M. Griffin, N. M. Trease, L. Trognko, P.-L. Taberna, P. Simon, and C. P. Grey, J. Am. Chem. Soc. 135, 18968 (2013); A. C. Forse, C. Merlet, J. M. Griffin, and C. P. Grey, ibid. 138, 5731 (2016).

[7] A. A. Kornyshev and R. Qiao, J. Phys. Chem. C 118, 18285 (2014).

[8] M. Z. Bazant, B. D. Storey, and A. A. Kornyshev, Phys. Rev. Lett. 106, 046102 (2011).

[9] C. Merlet, B. Rotenberg, P. A. Madden, P.-L. Taberna, P. Simon, Y. Gogotsi, and M. Salanne, Nat. Mater. 11, 306 (2012).

[10] A. Bankura, V. Carnevale, and M. L. Klein, J. Chem. Phys. 138, 014501 (2013).

[11] C. Merlet, D. T. Limmer, M. Salanne, R. van Roij, P. A. Madden, D. Chandler, and B. Rotenberg, J. Phys. Chem. C 118, 18291 (2014).

[12] A. Härtel, M. Janssen, S. Samin, and R. van Roij, J. Phys. Condens. Matter 27, 194129 (2015).

[13] G. Feng and P. T. Cummings, J. Phys. Chem. Lett. 2, 2859 (2011).

[14] P. Wu, J. Huang, V. Meunier, B. G. Sumpter, and R. Qiao, ACS Nano 5, 9044 (2011).

[15] D. E. Jiang, Z. Jin, and J. Wu, Nano Lett. 11, 5373 (2011).

[16] A. M. Smith, A. A. Lee, and S. Perkin, J. Phys. Chem. Lett. 7, 2157 (2016).

[17] M. Janssen, A. Härtel, and R. van Roij, Phys. Rev. Lett. 113, 268501 (2014).

[18] M. Janssen and R. van Roij, Phys. Rev. Lett. 118, 096001 (2017).

[19] J. Schiffer, D. Linzen, and D. U. Sauer, J. Power Sources 160, 765 (2006).

[20] J. Th. G. Overbeek, Colloids Surf. 51, 61 (1990).

[21] V. Kralj-Iglič and A. Iglič, J. Phys. II (France) 6, 477 (1996); P. M. Biesheuvel and M. van Soestbergen, J. Colloid Interface Sci. 316, 490 (2007).

[22] See Supplemental Material at http://link.aps.org/ supplemental/10.1103/PhysRevLett.119.166002, which includes Refs. [2,17,18,20,23-25], for a derivation of isothermal heat flow, the adiabatic temperature rise, the equality $\Pi_{\mathrm{rev}}^{\mathrm{ch}}=\Omega_{\mathrm{ent}}^{\mathrm{GC}}$, for the determination of the heat transfer coefficient and heat capacity, and for a discussion of the time scales of $I(t)$ and $\Delta T(t)$ decay in Fig. 3 .

[23] M. van Soestbergen, P. M. Biesheuvel, and M. Z. Bazant, Phys. Rev. E 81, 021503 (2010).

[24] F. P. Incropera, A. S. Lavine, T. L. Bergman, and D. P. DeWitt, Fundamentals of Heat and Mass Transfer (Wiley, New York, 2007).

[25] M. Z. Bazant, K. Thornton, and A. Ajdari, Phys. Rev. E 70, 021506 (2004).

[26] S. R. de Groot, Thermodynamics of Irreversible Processes (North-Holland, Amsterdam, 1951); S. R. de Groot and P. Mazur, Non-Equilibrium Thermodynamics (North-Holland, Amsterdam, 1962).

[27] P. M. Biesheuvel, D. Brogioli, and H. V. M. Hamelers, arXiv:1402.1448.

[28] A. L. d'Entremont and L. Pilon, J. Power Sources 246, 887 (2014). 